\title{
Evidence of downward trends in dental caries in Latin America and the Caribbean
}

\author{
What are the trends in dental caries prevalence and severity in Latin America \\ and the Caribbean?
}

\begin{abstract}
Bonecker M, Cleaton-Jones P. Trends in dental caries in Latin America and Caribbean 5-6 \& 11-13-yr-old children. A systematic review. Comm Dent Oral Epidemiol 2003; 31:152-157
\end{abstract}

Data sources Medline, Popline, ExtraMed, Embase and Info Latino Americo were searched for the period 1 January 1970-31 December 2000. Keywords used were "dental caries" and names of countries in Latin America and the Caribbean. Reference lists from retrieved articles were also examined for more studies.

Study selection For inclusion an article had to have clearly-defined ages within the groups (5-6, 11-13 years); use the World Health Organization caries diagnostic criteria; the sample had to be reasonably representative of the general population and contain at least 30 individuals; and examiners had to be calibrated.

Data extraction and synthesis For each of the two age-groupings, the caries prevalence and caries severity data were plotted against the year of study. A nonlinear regression curve was fitted to each group of results, and a linear regression analysis was done to look for a significant departure from zero of the caries-rate trend with statistical significance set at $P<0.05$. This was done separately for the 21 countries in Latin America and 12 countries in the Caribbean.

Results The search identified 438 articles: 172 were epidemiological studies, of which 30 fulfilled the inclusion criteria. A total of 16 countries were represented. Over the last 30 years, there has been a significant decrease in caries prevalence for 11-13-year-old children in Latin America. There was also a significant decrease in caries severity for 5-6year-olds in Latin America, and for 11-13-year-olds in Latin America and the Caribbean.

Conclusions This study showed some statistically significant downward trends in dental caries between 1970 and 2000 in 16 countries in Latin America and the Caribbean.

\section{Commentary}

This is a very important review which highlights the difficulties in comparing dental caries epidemiology within countries over time. The principal problem is comparing data for which there is little information available about the quality of the source study. As the authors point out, there is as yet no standard measure of study quality for observational studies.

The first point of note is that $56 \%$ (96 papers) of the epidemiological publications which may have been relevant were not included in the systematic review because they were difficult to locate. It is impossible to predict what impact these missing data would have made on the review conclusions (based upon 30 papers), and this should invoke caution in interpreting the results of the review.

Another interesting point is the problem of sampling to produce conclusions representative of a large country. In many countries there are wide geographical disparities in socio-economic status. A survey in one area in 1 year compared with other areas in subsequent years to produce dental caries trend information may be misleading. Unfortunately, as the authors highlight, information on sampling and methodology are often inadequate to allow reviewers to assess study quality appropriately.

In order to facilitate future systematic reviews in the area of dental caries epidemiology, further work is required on the standardisation of not only methodology but also for reporting.

\section{Gail Topping, Denise Furlanetto}

Dental Health Services Research Unit, University of Dundee, Dundee, Scotland, UK

Evidence-Based Dentistry (2004) 5, 68.

doi:10.1038/sj.ebd.6400279 